\title{
Building health impact assessment capacity as a lever for healthy public policy in urban planning
}

\author{
Jenny L. Hughes ${ }^{\mathrm{A}, \mathrm{C}}$ and Lynn A. Kemp ${ }^{\mathrm{B}}$ \\ ${ }^{\mathrm{A}}$ Centre for Chronic Disease Prevention and Health Advancement, \\ NSW Department of Health \\ ${ }^{\mathrm{B}} U N S W$ Research Centre for Primary Health Care and Equity, \\ University of New South Wales \\ ${ }^{\mathrm{C}}$ Corresponding author. Email: \\ jenny.hughes@doh.health.nsw.gov.au
}

\begin{abstract}
Building capacity to improve health through applying health impact assessment (HIA) increases the range of people, organisations and communities who are able to address health problems and, in particular, the problems that arise out of social inequity and social exclusion. To achieve this, a range of strategies is required across the areas of organisational development, workforce development, resource allocation, leadership and partnerships. A conceptual framework to guide understanding of capacity building evolved during a three-year capacity building project that supported the implementation of HIA. This is also applicable to the broader agenda of healthy public policy.
\end{abstract}

The NSW Health and Equity Statement ${ }^{1}$ recommended that processes should be developed for health impact assessment (HIA) as one set of strategies to ensure that proposed government policies, programs and projects would improve health and address health inequalities. ${ }^{2}$ In order to implement the Health and Equity policy statement, capacity to undertake HIA was required. Central to any capacity building approach is a clear perspective of whose capacity is to be built and the purpose of the capacity. This paper will describe the development of capacity building theory, ${ }^{3}$ which is needed to embed HIA as a viable tool for intersectoral action to improve considerations of health in urban planning.

That HIA is an effective tool for improving health in urban environments became apparent during a three-year project to build the capacity for HIA in NSW. To be effective, however, HIA needed to be embedded in both the health system and agencies other than health. Studies from other countries report difficulties in implementing healthy public policy and institutionalising HIA in a form that moves from a statement of intention to sustainable implementation. ${ }^{4-6}$ To embed HIA in NSW in an integrated and sustainable way required a range of strategies that were complementary to the capacity-building strategies used so far.

\section{Processes}

The NSW Health Impact Assessment Project ${ }^{2}$ began in 2002 with the intention of raising awareness, exploring the feasibility of the development of HIA in NSW and identifying areas where capacity needed to be built. In 2005, a three-year capacity building project commenced which aimed to integrate HIA into the NSW health system as a tool to improve internal planning and decision-making and as a way to engage external partners on initiatives that influence health outcomes.

Emerging in the early stages of the capacity building project was the realisation that the project was building capacity within the health system at a practitioner level. However, if HIA was to influence healthy urban planning, it also needed to be embedded in agencies other than NSW Health. This required additional development of infrastructure and sustainable intersectoral capacity.

To explore mechanisms to build this capacity a Healthy Urban Planning workshop was held in late 2005. Senior managers from NSW Health, the NSW Department of Planning and local government agencies attended the workshop. It aimed to build partnerships and capacity intersectorally. The workshop identified a limited organisational capacity not only within NSW Health but also in other agencies to work collaboratively and use HIA as a tool to strengthen current planning approaches. Also evident was that capacity needed to be built at different levels within organisations and that not all people in all systems need to have the skills to do an HIA.

A framework was developed that defined a set of capacitybuilding strategies at multiple levels (Figure 1). These became known as micro, meso and macro strategies to embed HIA in the health system and agencies other than health.

\section{Findings}

The capacity building project has demonstrated that to influence healthy urban planning, HIA capacity is needed in different ways at both operational and strategic levels. 
The micro strategies influence organisational capacity to implement HIA at an individual level. For example, the 'Learning by Doing' developmental site training program built the capacity of individuals and service teams to understand and implement the HIA methodology.

The meso strategies influence the agency commitment to providing resources and support that builds organisational capacity in HIA. Examples of that are: the Population Health Service Level Agreements between the Area Health Services and the NSW Department of Health, which stipulate a requirement to promote strategic alliances with agencies such as local government and to support the implementation of the NSW Health Impact Assessment Project.

The macro strategies have become the basis for the creation of healthy public policy approaches in agencies other than health to deliver program responses to health problems in a sustainable manner. An example is the creation of formal communication mechanisms between the NSW Department of Health and the Department of Planning on the use of HIA.

\section{Discussion}

Different types of HIA capacity need to be built at the micro, meso and macro levels of organisations in order to improve considerations of health in urban planning. A healthy public policy approach is useful for understanding these different levels of capacity. Healthy public policy improves the conditions under which people live: ie secure, safe, adequate and sustainable livelihoods. ${ }^{7}$

Making healthy public policy sustainable is multidimensional, requiring a range of strategies, at a range of levels that are continually tailored to opportunities arising from the development of individual and organisational capacity. Commonality of intent within individual systems to improve considerations of health in urban planning around policies, programs and projects to improve health may not always be enough.

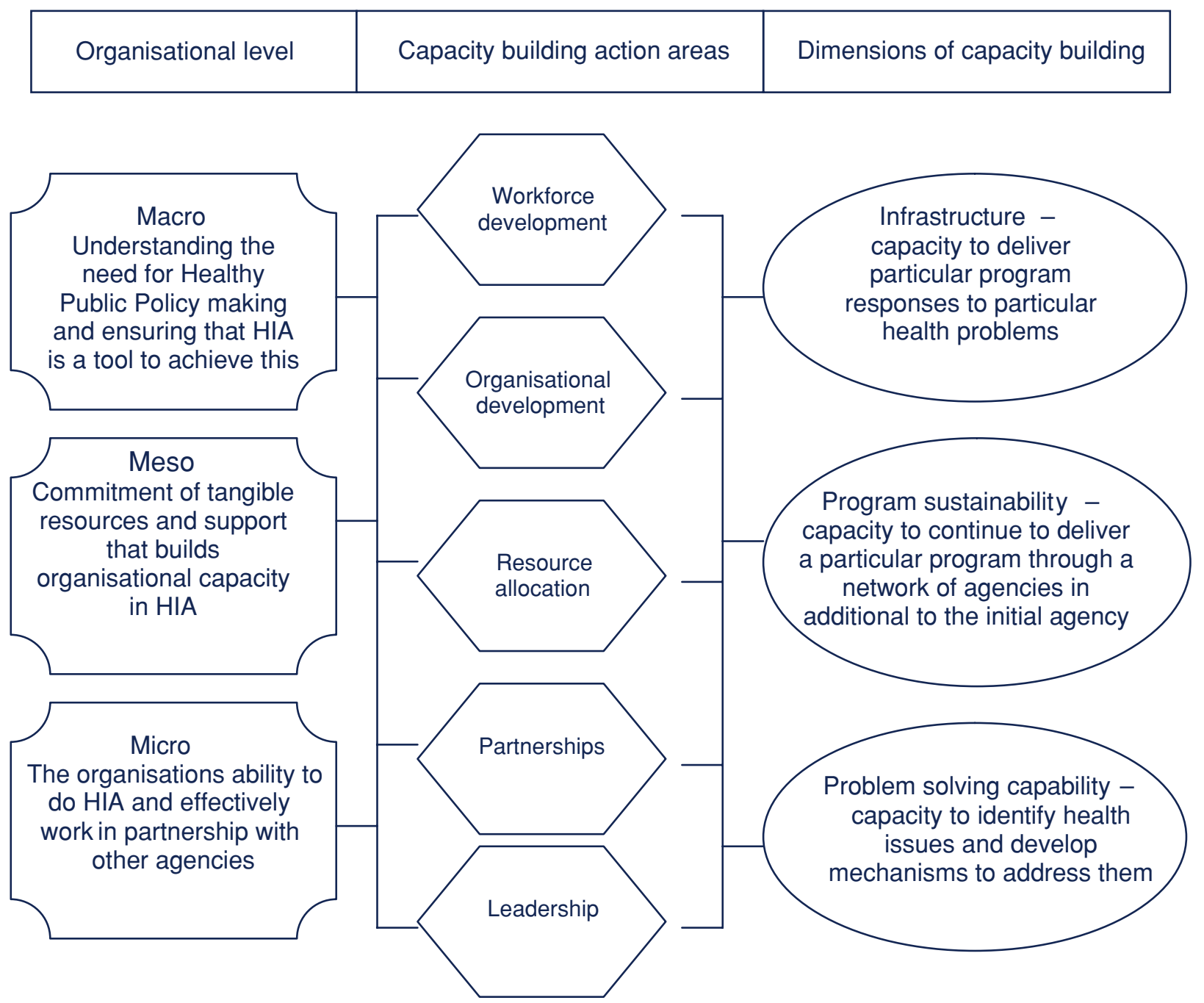

Figure 1. A framework for building capacity through multiple level strategies to embed health impact assessment and healthy public policy 
Sustainable intersectoral healthy public policy needs projects or programs based on this multidimensional aspect of capacity building. Multiple strategies at multiple levels need to be incorporated early in the planning process. In particular, consideration of strategies at the meso and macro level will support intersectoral outcomes that reflect joint infrastructure development, sustainability and organisations' problem-solving capability.

The HIA program is now therefore channelling efforts around decision-makers at the meso and macro level of health and human services to ensure that there is ongoing adoption of the social determinants of health as the basis for healthy urban planning and as a lever for the creation of healthy public policy approaches.

\section{References}

1. NSW Health and Equity Statement: in all fairness, increasing equity in health across NSW. Sydney: NSW Department of Health, 2004.
2. NSW Health Impact Assessment Project phase 1 project report. Sydney: Centre for Health Equity Training, Research and Evaluation, University of New South Wales, 2003.

3. A framework for building capacity to improve health. Sydney: NSW Department of Health, 2001. Available at www.health.nsw.gov.au, accessed 2 April 2007.

4. Gagnon F, Turgeon J, Dallaire C. Healthy public policy: a conceptual cognitive framework. Health Policy 2007; 81: 42-55. doi:10.1016/j.healthpol.2006.05.012

5. Mahoney M, Morgan R. Health impact assessment in Australia and New Zealand: an exploration of methodological concerns Int J Health Promot Educ 2001; 8(1): 8-11.

6. Harris E. NSW Health Impact Assessment Project review: a discussion paper prepared for the NSW HIA Steering Committee. Sydney: Research Centre for Primary Health Care and Equity, University of New South Wales, 2006.

7. Milio N. Glossary: healthy public policy. J Epidemiol Community Health 2001; 55: 622-3. http://jech.bmj.com/ cgi/content/full/55/9/622doi:10.1136/jech.55.9.622 